\title{
Molecular Profiling of Breast Carcinoma in Almadinah, KSA: Immunophenotyping and Clinicopathological Correlation
}

\author{
Mohamed A Elkablawy ${ }^{1,2 *}$, Abdelkader M Albasry ${ }^{1}$, Akbar S Hussainy ${ }^{1}$, Magdy \\ M Nouh ${ }^{1,3}$, Ahmed Alhujaily ${ }^{4}$
}

\begin{abstract}
Purpose: To subtype breast cancer (BC) in Saudi women according to the recent molecular classification and to correlate these subtypes with available clinicopathological parameters. Materials and Methods: Estrogen receptor (ER), progesterone receptor (PR) and human epidermal growth factor receptor (Her2/neu) immunostaining was semi-quantitatively assessed to define molecular subtypes of luminal A and B, HER-2 and triple negative (basallike) in BC paraffin embedded sections from 115 Saudi female patients diagnosed between 2005 to 2015 at the Department of Pathology, King Fahd Hospital, Almadinah, Saudi Arabia. Results: The most common subtypes were luminal A (47\%), followed by luminal $B(\mathbf{2 7 . 8 \%})$ and basal like subtypes $(\mathbf{1 8 . 3 \%})$, whereas HER-2 was the least common subtype (6.9\%). Luminal A was predominantly found in the old age group, with low tumor grade $(p<0.001)$ and small tumor size, whereas HER-2 and basal-like subtypes were significantly associated with young age, high tumor grade, lymph node metastasis and lymphovascular invasion $(p<0.03,0.004,0.05$ and 0.04 respectively). All subtypes showed advanced clinical stage at the time of presentation. Conclusions: Molecular subtypes of Saudi BC patients in Almadinah region are consistent with most of the worldwide subtyping. The biological behaviour of each molecular subtype could be expected based on its characteristic clinicopathological features. Along with other prognostic indicators, molecular subtyping would be helpful in predicting prognosis and management of our $\mathrm{BC}$ patients. We recommend screening and early diagnosis of $\mathrm{BC}$ in our population.
\end{abstract}

Keywords: Breast cancer - molecular - subtyping - ER - PR - Her2/neu - KSA

Asian Pac J Cancer Prev, 16 (17), 7819-7824

\section{Introduction}

Breast cancer $(\mathrm{BC})$ is one of the most common malignancies around the world (Ferlay et al., 2015). In the USA alone, there is an estimate of about a quarter of million new cases of BC cases during 2014, accounting for $14 \%$ of all new cancer cases (Howlader et al., 2013). Whereas, around 50285 new cases were diagnosed as $\mathrm{BC}$ in UK, accounts for $15 \%$ of all new cancer cases (Cancer Research UK, 2014). In the Kingdom of Saudi Arabia (KSA), the incidence of BC is much lower than in Western world i.e. only 1473 new cases were reported in 2010. However, $\mathrm{BC}$ is the most common malignancy in the Saudi women accounting for about one quarter of all the newly diagnosed female cancers (Saudi Cancer Registry, 2014). The presentation of BC in KSA is different from that in the Western countries as it has been reported to predominantly affect the younger population, commonly present as higher grades and in advanced stages (Alghamdi et al., 2013; Albasri et al., 2014).

$\mathrm{BC}$ is a disease of diversity, with regards to its natural history and treatment responses. Sometimes the traditional histological classification is unable to confine this diverse clinical heterogeneity of $\mathrm{BC}$. The prognosis and response to treatment are influenced by a number of factors such as histological grade, type and size of tumor, lymph node metastasis, estrogen receptor (ER), progesterone receptor (PR) and human epidermal growth factor receptor 2 (Her-2/neu) status. Thus the recent BC research has now diverted from histological classification towards molecular classification. Development of new classifications based on immunohistochemical, genetic and molecular findings are subsequently in evolution (Pusztai et al., 2006; Pusztai, 2008).

The history of molecular classification dates back to about 30 years with the availability of hormone (estrogen and progesterone) receptor markers. This was followed by the emergence of Her-2/neu determination techniques. It was only 15 years back, that a group of scientists from California, came up with the molecular classification of human BC, initially divided BC into four major classes: luminal-like, basal-like, normal-like, and HER-2 positive 
(Perou et al., 2000). Consequently a fifth class was added, dividing the luminal class to luminal $\mathrm{A}$ and luminal $\mathrm{B}$ classes (Carey et al., 2006).

As the presentation of $\mathrm{BC}$ in $\mathrm{KSA}$ is unlike that reported from the western literature, the aim of our study is to subtype our cases of $\mathrm{BC}$ according to the recent molecular classification on the basis of ER, PR and Her-2/ neu receptor status on the paraffin blocks; and to correlate with available clinicopathological parameters.

\section{Materials and Methods}

\section{Patient's tissue and histopathological evaluation}

A total of 115 female patients histopathologically confirmed as primary invasive BC were enrolled in this study. Cases were collected from the department of pathology, King Fahd hospital, Almadinah, KSA, between January 2005 and March 2015. All cases were reviewed by two of the authors (A.A.\& M.E.) and detailed clinical and histopathological data including patient's age, tumor size, lymph node invasion and lymphovascular status were collected (Table 1). Histologic grade was assessed according to a Modified Bloom-Richardson Scoring system (MBR). In-situ lesions, recurrences, biopsies, sarcomas, benign and secondary lesions were excluded from the study. Modified radical mastectomies, quadrectomy and wide local excision specimens with axillary surgery were included. Bilateral BC were regarded individually on the basis of the characteristics of each cancer.

\section{Immunohistochemical staining}

Tissue cores were extracted from archival blocks of the primary $\mathrm{BC}$ and used to construct a tissue miniarray (TmA) as previously described (Elkablawy and Albasri, 2015). IHC staining of ER, PR and Her-2/neu was carried out by using Ventana Bench Mark XT system (Ventana Medical Systems, Tucson, AZ). All procedures were performed automatically in the Bench Mark. IHC staining was performed on 4- $\mu \mathrm{m}$ slices of formalin-fixed paraffinembedded (FFPE) TmA tissue sections with primary antibodies against ER (SP1, 1:100, Dako, Denmark), PR (PgR 636, 1:100, Dako, Denmark) and Her-2/neu (4B5, Roche, Switzerland). The tissue sections were incubated with primary antibody of ER, and PR for 32 minutes at $42^{\circ} \mathrm{C}$ and of Her- $2 /$ neu for 16 minutes at $42^{\circ} \mathrm{C}$. The avidinbiotin technique was performed using diaminobenzidin (DAB) for visualization and sections were counterstained with hematoxylin.

\section{Immunoscoring and molecular subtyping of $B C$}

Positivity for ER or PR was documented when any nuclear staining was evidenced, according to the St Gallen consensus 2009 (Goldhirsch et al., 2009). Tumors that showed strong complete membranous staining in $>$ $10 \%$ cells were taken as positive for Her- $2 /$ neu. Based on combined immunoprofile data of ER, PR and Her-2/neu, $\mathrm{BC}$ were classified into subtypes as follows: luminal A (ER+, PR+/-, Her-2/neu -), luminal B (ER+, PR+/-, Her2/neu +), HER-2 (ER-, PR-, Her-2/neu +), and basal-like (ER-, PR-, Her-2/neu -).

\section{Statistical evaluation:}

All statistical analyses were carried out in SPSS version 22.0 (SPSS, Inc., Chicago, IL) with two-tailed test. Statistical analysis was assessed using chi-square test and fisher exact test. $\mathrm{P} \leq 0.05$ was considered statistically significant.

\section{Results}

\section{Clinicopathological distinctive features}

Detailed clinical and histopathological features of the 115 female BC patients included in this study are shown in Table 1 . The mean age was 50 years (range from 26 to 88 years). About half of the patients (50.4\%) were the younger age group ( $\leq 50$ years). Right breast was involved in $(47.8 \%)$, left breast in $(44.2 \%)$ and $8 \%$ cases had bilateral involvement. The most common morphological category was infiltrating ductal carcinoma $(94.9 \%)$, whereas other types included infiltrating lobular carcinoma, mucinous carcinoma and metaplastic

Table 1. Correlation of Clinical and Histopathological Parameters with the molecular subtypes of Breast Cancer in Our study

\begin{tabular}{|c|c|c|c|c|c|c|}
\hline Parameters & & $\begin{array}{l}\text { Luminal A } \\
\text { Subtype }\end{array}$ & $\begin{array}{l}\text { Luminal B } \\
\text { Subtype }\end{array}$ & $\begin{array}{l}\text { Basal-like } \\
\text { Subtype }\end{array}$ & HER-2 Subtype & $\mathrm{P}$ \\
\hline Distribution & $54(47 \%)$ & $32(27.8 \%)$ & $21(18.3 \%)$ & $8(6.9 \%)$ & & \\
\hline \multirow[t]{2}{*}{ Age (years) } & $\leq 50$ & $25(46.3 \%)$ & $11(34.4 \%)$ & $16(76.2 \%)$ & $6(75 \%)$ & $0.03 *$ \\
\hline & $>50$ & $29(53.7 \%)$ & $21(65.6 \%)$ & $5(23.8 \%)$ & $2(25 \%)$ & \\
\hline \multirow[t]{3}{*}{ Tumor grade } & Grade I & $5(9.3 \%)$ & $1(3.1 \%)$ & 0 & 0 & $0.004 *$ \\
\hline & Grade II & $34(62.9 \%)$ & $11(34.4 \%)$ & $12(57.1 \%)$ & $1(12.5 \%)$ & \\
\hline & Grade III & $15(27.8 \%)$ & $20(62.5 \%)$ & $9(42.9 \%)$ & $7(87.5 \%)$ & \\
\hline \multirow[t]{3}{*}{ Tumor size } & $\mathrm{T} 1:<2 \mathrm{~cm}$ & $1(2.3 \%)$ & $2(6.3 \%)$ & $5(23.8 \%)$ & 0 & 0.13 \\
\hline & $\mathrm{T} 2: 2-5 \mathrm{~cm}$ & $38(86.4 \%)$ & $25(78.1 \%)$ & $14(66.7 \%)$ & $8(100 \%)$ & \\
\hline & $\mathrm{T} 3:>5 \mathrm{~cm}$ & & $5(11.3 \%)$ & $5(15.6 \%)$ & $2(9.5 \%)$ & 0 \\
\hline \multirow[t]{4}{*}{ LN metastases } & Negative & $23(42.5 \%)$ & $6(18.7 \%)$ & $8(38.1 \%)$ & $1(12.5 \%)$ & $0.05 *$ \\
\hline & Positive & & & & & \\
\hline & 1-3 LN & $6(11.1 \%)$ & $8(25 \%)$ & $3(14.3 \%)$ & $1(12.5 \%)$ & \\
\hline & $>3 \mathrm{LN}$ & $25(46.4 \%)$ & $18(56.3 \%)$ & $10(47.6 \%)$ & $6(75 \%)$ & \\
\hline \multirow[t]{2}{*}{ Lymphovascular invasion } & Negative & $21(38.9 \%)$ & $7(21.8 \%)$ & $4(19.1 \%)$ & 0 & $0.04 *$ \\
\hline & Positive & $33(61.1 \%)$ & $25(78.2 \%)$ & $17(80.9 \%)$ & $8(100 \%)$ & \\
\hline
\end{tabular}

*Significant at $\mathrm{P} \leq 0.05$ 
carcinoma accounted for 2 cases each (1.7\%). Most tumors were of intermediate (grade II; 50.4\%) and high grades (grade III; 44.4\%). Mean tumor size was $3.38 \mathrm{~cm}$ (range from 0.5 to $12 \mathrm{~cm})$. Seventy four point eight $(74.8 \%)$ of cases were between $2-5 \mathrm{~cm}, 15.7 \%$ were smaller $<2$ $\mathrm{cm}$, and $9.5 \%$ were larger $>5 \mathrm{~cm}$. Eighty three patients $(72.2 \%)$ had lymphovascular invasion, while $77(67 \%)$ were associated with positive lymph nodes. The majority $(51.3 \%)$ of which had metastasis in more than $3 \mathrm{LN}$ and (15.6\%) had metastases in 1-3 LN.

\section{Immunoprofiles and subtyping}

Individual Immunoprofile analysis revealed a higher PR and ER positivity levels (62.7\% and 61.8\%) in BC cases respectively, and Her-2/neu over expression in (38.2\%) of BC. Based on the combined immunoprofile results of these biomarkers, the highest frequency of BC subtypes were luminal A (Figure 1) accounting for $47 \%$ cases, followed by luminal B $(27.8 \%)$, and basal-like subtype (18.3\%). Whereas HER-2 (Figure 2) was the least common $(6.9 \%)$ subtype (Table 1$)$.

\section{BC Subtypes distinctive features and statistical associations}

Table 1 shows the detailed correlation of BC molecular subtypes and different clinicopathological features. The mean age of luminal A subtype was 50.3 years. Most of the cases were moderately differentiated $(62.9 \%)$, but the majority of well differentiated tumors of our study were found also in this molecular subtype $(9.3 \%)$. We observed that tumors of small dimensions (T1-T2) were predominant in this subtype (92.6\%). Axillary lymph node metastases were present in $57.5 \%$ and lymphovascular invasion (LVI) was found in $61.1 \%$ of cases.

In luminal B subtype, the mean age was 54.9 years. Most of the cases were poorly and moderately differentiated carcinomas (62.5\% and 34.4\% respectively). Tumors of large dimensions (T2-T3) constituted (93.7\%) of the cases in luminal B subtype. Axillary lymph node metastasis was present in $81.3 \%$ and LVI was found in $78.2 \%$ of cases.

In contrast to luminal $\mathrm{A}$ and luminal $\mathrm{B}$, basal-like subtype occurred in younger age group with a mean age of 48.4 years. The most frequent tumor grade was grade II $(57.1 \%)$ and grade III (42.9\%). $76.2 \%$ of the cases with basal-like subtype presented with tumors of large dimensions (T2-T3). Axillary lymph node metastasis was present in $61.9 \%$ and lymphovascular invasion was found in $80.9 \%$ of cases.

HER-2 subtype was the most frequent molecular subtype in younger age group with a mean age of 46.4 years. Almost all cases were poorly differentiated (87.5\%) and had axillary lymph node metastasis in $87.5 \%$ cases. LVI was found in all cases.

Statistical analysis showed a significant difference regarding the molecular subtypes of BC in our study against tested clinicopathological parameters (Table 1). Her-2 and Basal-like subtypes occurred more frequently in younger $(\leq 50$ yrs age $)$ patients group $(\mathrm{P}=0.03)$ compared to luminal A and B subtypes. The highest frequency of nodal positivity ( $>3 \mathrm{LN}$ ) was associated with HER-2 overexpressing $\mathrm{BC}$, followed by luminal $\mathrm{B}$ subtype $(\mathrm{P}=$
Table 2. Correlation of age with Immunoprofiles Data and Clinicopathological Parameters of BC Cases

\begin{tabular}{|c|c|c|c|}
\hline Variable & $\begin{array}{l}\text { Young age group } \\
\text { ( } \leq 50 \text { years old })\end{array}$ & $\begin{array}{l}\text { Old age group } \\
(>50 \text { years old) }\end{array}$ & $\mathrm{p}$ value \\
\hline \multicolumn{4}{|l|}{ Tumor grade } \\
\hline Grade I & 2 & 4 & 0.022 \\
\hline Grade II & 23 & 35 & \\
\hline Grade III & 33 & 18 & \\
\hline \multicolumn{4}{|l|}{ Tumor size } \\
\hline $\mathrm{T} 1:<2 \mathrm{~cm}$ & 8 & 10 & 0.755 \\
\hline $\mathrm{T} 2: 2-5 \mathrm{~cm}$ & 43 & 42 & \\
\hline T3: $>5 \mathrm{~cm}$ & 8 & 4 & \\
\hline \multicolumn{4}{|l|}{ LN metastases } \\
\hline Negative & 14 & 24 & 0.121 \\
\hline $1-3 \mathrm{LN}$ & 10 & 8 & \\
\hline$>3 \mathrm{LN}$ & 34 & 25 & \\
\hline \multicolumn{4}{|c|}{ Lymphovascular invasion } \\
\hline Negative & 11 & 21 & 0.041 \\
\hline Positive & 47 & 36 & \\
\hline \multicolumn{4}{|c|}{ Estrogen receptor status } \\
\hline Negative & 29 & 15 & 0.014 \\
\hline Positive & 29 & 42 & \\
\hline \multicolumn{4}{|c|}{ Progesterone receptor status } \\
\hline Negative & 28 & 15 & 0.025 \\
\hline Positive & 30 & 42 & \\
\hline \multicolumn{4}{|l|}{ Her- $2 /$ neustatus } \\
\hline Negative & 33 & 38 & 0.377 \\
\hline Positive & 25 & 19 & \\
\hline \multicolumn{4}{|c|}{ Molecular subtypes } \\
\hline Luminal A & 25 & 29 & 0.011 \\
\hline Luminal B & 11 & 21 & \\
\hline Basal-like & 16 & 5 & \\
\hline Her-2/neu & 6 & 2 & \\
\hline
\end{tabular}

*Significant at $\mathrm{P} \leq 0.05$

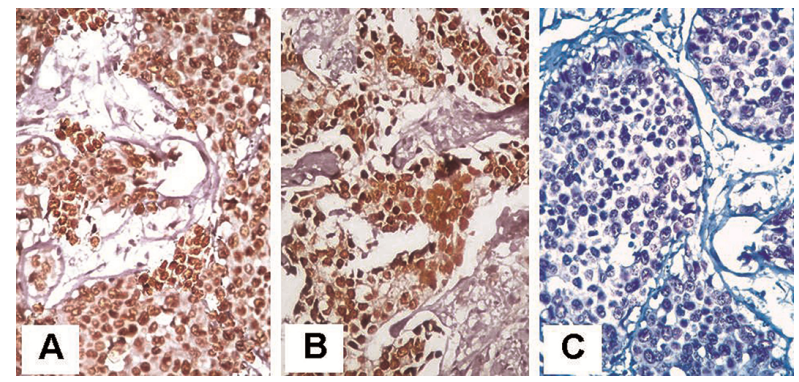

Figure 1. Breast Invasive Duct Carcinoma, Luminal A Subtype Showing Positive Nuclear Immunostaining for ER (A) and PR (B), whereas Negative for Her-2/ neu (C). Magnification $x 400$

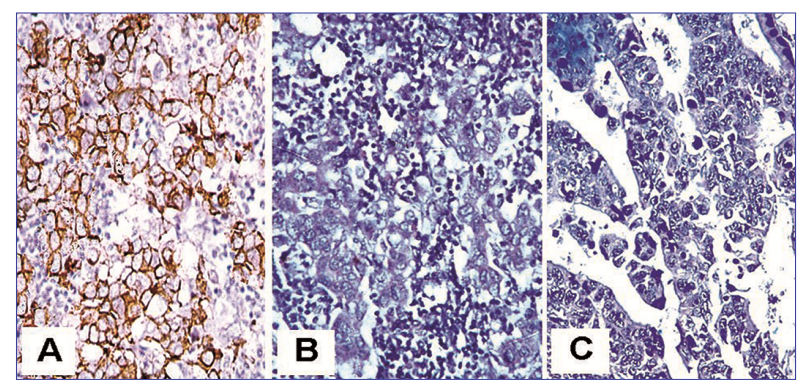

Figure 2. Breast iInvasive Duct carcinoma, HER-2 subtype showing positive Membranous immunostaining for Her-2/neu (A), negative nuclear staining for ER (B) and PR (C). Magnification x400 
Table 3. Proportions of Various Molecular Subtypes of BC as Reported in the Recent Medical Literature

\begin{tabular}{|c|c|c|c|c|c|c|}
\hline Study & Year & Country & Luminal A (\%) & Luminal B (\%) & HER $2(\%)$ & TN/Basal (\%) \\
\hline Bhargava et al & 2009 & USA & 55 & 17 & 4 & 15 \\
\hline Ben Abdelkrim et al & 2010 & Tunis & 51.5 & 16 & 14.5 & 18 \\
\hline Tamimi et al & 2010 & $\mathrm{KSA}$ & 3.9 & 16 & 17.3 & 10 \\
\hline Shibuta et al & 2011 & Japan & 71 & 8 & 9 & 12 \\
\hline Saliha et al & 2011 & Egypt & 44.3 & 24.6 & 11.8 & $11.3[+(7.9 \%)]^{*}$ \\
\hline El-Hawary et al & 2012 & Egypt & 41.2 & 13.9 & 19.4 & 28.5 \\
\hline Elesawy et al & 2012 & Egypt & 39.2 & 14.4 & 13.6 & $16.8[+(16 \%)]^{*}$ \\
\hline Shomaf et al & 2013 & Jordan & 60 & 13 & 12 & 15 \\
\hline Caldarella et al & 2013 & Italy & 34 & 36 & 10.2 & 19 \\
\hline Zhu et al & 2014 & China & 65.3 & 19 & 6.5 & 9.2 \\
\hline Zheng et al & 2014 & China & 54.2 & 14 & 8.9 & 22.9 \\
\hline Akbar et al & 2014 & Pakistan & 28.3 & 25 & 30 & 16.7 \\
\hline Cherbal et al & 2015 & Algerian & 50.59 & 19.67 & 8.92 & 20.8 \\
\hline Our Study & 2015 & KSA & 47 & 27.8 & 6.9 & 18.3 \\
\hline
\end{tabular}

* Unclassified group

0.05). Moreover they also had tendency to show higher tumor grade $(\mathrm{III})$ at presentation $(\mathrm{P}=0.004)$. Positive LVI was prevailed in HER-2 followed by basal-like and luminal $\mathrm{B}$ subtypes $(\mathrm{P}=0.04)$. However, there was no significant difference in tumor size among any subtype $(\mathrm{P}=0.13)$.

Statistical effects of age grouping on different variables

Correlations of the two age groups with other variables are shown in Table 2. In comparison to old age group, young age group patients presented with high grade tumors $(\mathrm{p}<0.02)$, LVI $(\mathrm{p}<0.04)$, low ER and PR expression ( $p$ $<0.01$ and $p<0.02$ ) respectively. However, no significant association was found between the two groups with regards to tumor size, lymph node metastasis and Her-2/ neu expression. Based on molecular sub classification, we found a significantly increased proportion of basallike and HER-2 subtypes in young age group compared to old age group $(27.5 \%$ vs.8.7\% and $10.3 \%$ vs.3.5\% respectively; $\mathrm{P}<0.01)$.

\section{Discussion}

In the present study, we have investigated the frequency of individual and combined molecular subtypes of BC in a cohort of 115 Saudi women, based on IHC of ER, PR and Her-2/neu as a well-established method for classification (Pusztai et al., 2006; Pusztai, 2008). The immunostaining results of ER, PR and Her-2/neu in this study are in agreement with similar recent studies (Andrade et al., 2014; Elesawy et al., 2014). However a variable difference does exist with others (Bhargava et al., 2009; Cheang et al., 2009). These observations suggest that ER, PR and HER-2/neu expression in BC around the world vary considerably among different populations, probably due to racial and genetic differences.

In our study, we found luminal A to be the most common molecular subtype. This finding is consistent with most worldwide reports from USA, Europe, Asia and Africa (Bhargava et al., 2009; Salhia et al., 2011; Zhu et al., 2014; Cherbal et al., 2015). As reported in many studies (Bhargava et al., 2009; Salhia et al., 2011; Zhu et al., 2014), luminal B was the second common subtype among our cohort. In contrast, our findings are not in keeping with results of some studies from Japan, Tunis and Jordan (Abdelkrim et al., 2010; Shibuta et al., 2011; Shomaf et al., 2013). The findings from some of the recent studies are compared in Table 3 . As observed, in our study the percentage of triple negative / basal subtype was represented by $15-20 \%$ as in many of the available studies in medical literature (Bhargava et al., 2009; Caldarella et al., 2013; Shomaf et al., 2013; Elesawy et al., 2014; Cherbal et al., 2015). In contrast, a higher percentage between (22.9 -32\% was reported in studies from China, Egypt and Nigeria (Huo et al., 2009; El-Hawary et al., 2012; Zheng et al., 2014). Regarding HER-2 subtype, the results from China and Algeria are consistent with our findings (Zhu et al., 2014; Cherbal et al., 2015). Whereas researchers from Pakistan have reported very high figures of HER-2 subtype (Akbar et al., 2014). In contrast most of studies from the developed world show low figures of HER-2 subtype (Bhargava et al., 2009; Shibuta et al., 2011; Caldarella et al., 2013). These variable ethnic differences in $\mathrm{BC}$ subtypes could be due to an aetiological heterogeneity of $\mathrm{BC}$ and different genetic background.

In the present study, in addition to classifying the $\mathrm{BC}$ cases into four molecular subtypes, we also correlated each subtype with the available clinicopathological parameter including age, tumor grade, tumor size, lymph node metastasis and LVI. Our work supports the well established aggressiveness of basal-like (triple negative) and HER2 tumors, as these two subtypes were more frequent in younger age group, as reported in recent literature (Akbar et al., 2014; Kruijf et al., 2014); as well as presented with higher grades and poor prognostic parameters; in keeping with recent researches from Algeria and Italy (Caldarella et al., 2013; Cherbal et al., 2015). The Japanese researchers have also highlighted this phenomenon in a different way by concluding that the HER-2 positive subtypes (luminal B and HER-2 subtypes) had a significantly higher incidence of lymph node metastasis and lymphatic permeation, while the hormone receptor negative subtypes (HER-2 and TN subtypes) showed a significantly higher nuclear grade (Shibuta et al., 2011). Whereas our observation regarding the two less aggressive luminal subtypes, are that these occurred in older age group, were found to be better differentiated (Grade I and II) and of smaller size. These findings are consistent with observations already 
reported in the literature (Shomaf et al., 2013; Zheng et al., 2014). An important point of exception was significant percentages of lymph node metastasis and LVI in both luminal subtypes, which is probably due to advanced BC stage at the time of presentation in all the subtypes in our population (Alghamdi et al., 2013). This fact necessitates more organized efforts to highlight the importance of expanding our public awareness regarding BC screening programs and early diagnosis.

When the findings were adjusted for age, our study revealed significant differences in tumor grade, LVI and low hormonal receptor status, which have also been recently reported from China (Zheng et al., 2014). We found triple negative more common in younger age group in keeping with studies from Netherland (Kruijf et al., 2014). In contrast a study from Brazil found significantly increased rates of triple negative tumors, in patients above 50 years age (Andrade et al., 2014). Thus there are wide variations from different regions and populations with regards to the prevalence of the molecular subtypes, probably due to genetic reasons or methodology of studies and calculation. However we can emphasize that this comparison of the two age groups in our study points out towards the fact that BC in our young group patients, present with poor prognostic parameters such as low hormonal (ER \& PR) status, high HER-2/neu status, high tumor grade, lymph node metastasis and LVI.

In conclusion, we found that molecular subtypes of Saudi BC patients in Almadinah are consistent with most of the worldwide reports with minor differences. The biological behavior of each molecular subtype could be expected based on its characteristic clinicopathological features. Along with other prognostic indicators, molecular subtyping would thus be helpful in predicting prognosis and management of our Saudi BC patients. BC in our young group patients, present with poor prognostic parameters, thus we recommend increasing the public awareness regarding the importance of self examination, screening programs and early diagnosis of BC.

\section{Acknowledgements}

The authors thank the Deanship of Scientific research of Taibah University, Almadinah Almonawwarah, KSA, for their encouragement and financial support. The authors thank Mr. Mohamed Abdelsammad (Microbiology Lab., Taibah University, KSA), Mrs Faten (Pathology Lab., Taibah University, KSA) and Mr. Khalid Salam (Pathology Lab., Menoufia University, Egypt) for technical advice.

\section{References}

Akbar M, Akbar K, Naveed D (2014). Frequency and correlation of molecular subtypes of breast cancer with clinicopathological features. J Ayub Med Coll Abbottabad, 26, 290-3.

Al Tamimi DM, Shawarby MA, Ahmed A, et al (2010). Protein expression profile and prevalence pattern of the molecular classes of breast cancer - a Saudi population based study. BMC Cancer, 10, 223-35.

Albasri A, Hussainy AS, Sundkji I, et al (2014). Histopathological features of breast cancer in Al-Madinah region of Saudi
Arabia. Saudi Med J, 35, 1489-93.

Alghamdi IG, Hussain II, Alghamdi MS, et al (2013). The incidence rate of female breast cancer in Saudi Arabia: an observational descriptive epidemiological analysis of data from Saudi Cancer Registry 2001-2008. Breast Cancer (Dove Med Press), 5, 103-9.

BenAbdelkrim S, Trabelsi A, Missaoui N, et al (2010). Distribution of molecular breast cancer subtypes among Tunisian women and correlation with histopathologicalparameters: a study of 194 patients. Pathol Res Pract, 206, 772-5.

Bhargava R, Striebel J, Beriwal S, et al (2009). Prevalence, morphologic features and proliferation indices of breast carcinoma molecular classes using immunohistochemical surrogate markers. Int J Clin Exp Pathol, 2, 444-55.

Caldarella A, Buzzoni C, Crocetti E, et al (2013). Invasive breast cancer: a significant correlation between histological types and molecular subgroups. J Cancer Res Clin Oncol, 139, 617-23.

Cancer Research UK (2014): Breast cancer incidence statistics 2011 [Online]. Cancer Research UK. Available: http://www. cancerresearchuk.org/cancer-info/cancerstats/types/breast/ incidence/uk-breast-cancer-incidence-statistics. [Accessed on 28 January 2015].

Carey LA, Perou CM, Livasy CA, et al (2006). Race, breast cancer subtypes, and survival in the Carolina Breast Cancer Study. JAMA, 295, 2492-502.

Cheang MC, Chia SK, Voduc D, et al (2009). Ki67 index, HER2 status, and prognosis of patients with luminal B breast cancer. $J$ Natl Cancer Inst, 101, 736-50.

Cherbal F, Gaceb H, Mehemmai C, et al (2015). Distribution of molecular breast cancer subtypes among Algerian women and correlation with clinical and tumor characteristics: A population-based study. Breast Dis, 35, 95-102.

de Kruijf EM, Bastiaannet E, Rubertá F, et al (2014). Comparison of frequencies and prognostic effect of molecular subtypes between young and elderly breast cancer patients. $\mathrm{Mol}$ Oncol, 8, 1014-25.

de Macêdo Andrade AC, Ferreira Júnior CA, Dantas Guimarães $B$, et al (2014). Molecular breast cancer subtypes and therapies in a public hospital of Northeastern Brazil. BMC Women's Health, 14, 110-18.

Elesawy BH, Abd El hafez A, Shawky Ael-A, et al (2014). Immunohistochemistry-based subtyping of breast carcinoma in Egyptian women: a clinicopathologic study on 125 patients. Ann Diagn Pathol, 18, 21-6.

El-Hawary AK, Abbas AS, Elsayed AA, et al (2012). Molecular subtypes of breast carcinoma in Egyptian women: clinicopathological features. Pathol Res Pract, 208, 382-6.

Elkablawy MA, Albasri AM (2015). High quality tissue miniarray technique using a conventional TV/Radio telescopic antenna. Asian Pac J Cancer Prev, 16, 1129-133.

Ferlay J, Soerjomataram I, Ervik M, et al (2015). Cancer incidence and mortality worldwide: sources, methods and major patterns in GLOBOCAN 2012. Int J Cancer, 136, 359-86.

Goldhirsch A, Ingle JN, Gelber RD, et al (2009). Thresholds for therapies: highlights of the St Gallen International Expert Consensus on the primary therapy of early breast cancer 2009. Ann Oncol, 20, 1319-29.

Howlader N, Noone AM, Krapcho M, et al (2013). SEER Cancer Statistics Review, 1975-2011, National Cancer Institute. Bethesda, MD, http://seer.cancer.gov/csr/1975_2011/,based on November 2013 SEER data submission, posted to the SEER web site, April 2014.

Huo D, Ikpatt F, Khramtsov A, et al (2009). Population differences in breast cancer: survey in indigenous African women reveals over-representation of triple-negative breast 
Mohamed A Elkablawy et al

cancer. J Clin Oncol, 27, 4515-21.

Perou CM, Sorlie T, Eisen MB, et al (2000). Molecular portraits of human breast tumours. Nature, 406, 747-52.

Pusztai L, Mazouni C, Anderson K, et al (2006). Molecular classification of breast cancer: limitations and potential. Oncologist, 11, 868-77.

Pusztai L (2008). Current status of prognostic profiling in breast cancer. Oncologist, 13, 350-60.

Salhia B, Tapia C, Ishak EA, et al (2011). Molecular subtype analysis determines the association of advanced breast cancer in Egypt with favorable biology. BMC Womens Health, 11, 44-52.

Saudi Cancer Registry (2014). Cancer incidence report Saudi Arabia 2010 [Online]. Saudi Cancer Registry. Available: http://www.scr.org.sa. [Accessed on 26 January 2015].

Shibuta K, Ueo H, Furusawa H, et al (2011). The relevance of intrinsic subtype to clinicopathological features and prognosis in 4,266 Japanese women with breast cancer. Breast Cancer, 18, 292-8.

Shomaf M, Masad J, Najjar S, et al (2013). Distribution of breast cancer subtypes among Jordanian women and correlation with histopathological grade: molecular subclassification study. JRSM Short Rep, 4, 1-6.

Zheng S, Song QK, Ren Y, et al (2014). The characteristics of breast cancer subtypes: Implications for treatment guidelines and individualized treatment strategies in China. Appl Immunohistochem Mol Morphol, 22, 383-9.

Zhu X , Ying J, Wang F, et al (2014). Estrogen receptor, progesterone receptor, and human epidermal growth factor receptor 2 status in invasive breast cancer: a 3,198 cases study at National Cancer Center, China. Breast Cancer Res Treat, 147, 551-5. 